\title{
Symptoms of generalized anxiety disorder but not panic disorder at age 15 years increase the risk of depression at 18 years in the Avon Longitudinal Study of Parents and Children (ALSPAC) cohort study
}

\author{
S. J. C. Davies ${ }^{1,2 *}$, R. M. Pearson ${ }^{1}$, L. Stapinski ${ }^{3}$, H. Bould ${ }^{1}$, D. M. Christmas ${ }^{1,4}$, K. S. Button ${ }^{1}$, \\ P. Skapinakis ${ }^{1}$, G. Lewis ${ }^{5}$ and J. Evans ${ }^{1}$ \\ ${ }^{1}$ Centre for Academic Mental Health, School of Social and Community Medicine, University of Bristol, Bristol, UK \\ ${ }^{2}$ Geriatric Psychiatry Division, CAMH, University of Toronto, Toronto, Canada \\ ${ }^{3}$ NHMRC Centre for Research Excellence in Mental Health and Substance Use, National Drug and Alcohol Research Centre, University of New \\ South Wales, Sydney, Australia \\ ${ }^{4}$ Cambridgeshire and Peterborough NHS Foundation Trust, Cambridge, UK \\ ${ }^{5}$ Division of Psychiatry, University College London, London, UK
}

Background. Generalized anxiety disorder (GAD) and panic disorder (PD) differ in their biology and co-morbidities. We hypothesized that GAD but not PD symptoms at the age of 15 years are associated with depression diagnosis at 18 years.

\begin{abstract}
Method. Using longitudinal data from the Avon Longitudinal Study of Parents and Children (ALSPAC) birth cohort we examined relationships of GAD and PD symptoms (measured by the Development and Well-Being Assessment) at 15 years with depression at 18 years (by the Clinical Interview Schedule - Revised) using logistic regression. We excluded adolescents already depressed at 15 years and adjusted for social class, maternal education, birth order, gender, alcohol intake and smoking. We repeated these analyses following multiple imputation for missing data.
\end{abstract}

Results. In the sample with complete data $(n=2835)$, high and moderate GAD symptoms in adolescents not depressed at 15 years were associated with increased risk of depression at 18 years both in unadjusted analyses and adjusting for PD symptoms at 15 years and the above potential confounders. The adjusted odds ratio (OR) for depression at 18 years in adolescents with high relative to low GAD scores was 5.2 [95\% confidence interval (CI) 3.0-9.1, overall $p<0.0001]$. There were no associations between PD symptoms and depression at 18 years in any model (high relative to low PD scores, adjusted $\mathrm{OR}=1.3,95 \% \mathrm{CI} 0.3-4.8$, overall $p=0.737$ ). Missing data imputation strengthened the relationship of GAD symptoms with depression (high relative to low GAD scores, OR =6.2, 95\% CI 3.9-9.9) but those for PD became weaker.

Conclusions. Symptoms of GAD but not PD at 15 years are associated with depression at 18 years. Clinicians should be aware that adolescents with GAD symptoms may develop depression.

Received 22 June 2014; Revised 6 July 2015; Accepted 8 July 2015; First published online 28 August 2015

Key words: Adolescents, anxiety, Avon Longitudinal Study of Parents and Children, depression, generalized anxiety disorder.

\section{Introduction}

Depression is a leading cause of morbidity with a high cost to society (Sobocki et al. 2006). The incidence of depression increases rapidly during late adolescence. For example in the Dunedin study (Jaffee et al. 2002) the prevalence rose from $4 \%$ at age 15 years to $16 \%$ at

* Address for correspondence: S. J. C. Davies, Geriatric Psychiatry Division, Centre for Addiction and Mental Health (CAMH), 6th Floor, 80 Workman Way, Toronto, Ontario M6J 1H4, Canada.

(Email: simon_davies@camh.net) age 18 years, while in the National Comorbidity Survey adolescent supplement the prevalence of major depression or dysthymia rose from $8.7 \%$ at age 13-14 years to $15.7 \%$ at age $17-18$ years (Merikangas et al. 2010). Depression with onset during adolescence carries a high risk of relapse, for example in one study in the USA, $40 \%$ of 16 year olds with depression experienced a relapse by the age of 23 years (Lewinsohn et al. 1999). In addition, adolescence is an important period for the acquisition of education and development of social and interpersonal skills and a period of depression may disrupt these formative

This is an Open Access article, distributed under the terms of the Creative Commons Attribution licence (http://creative commons.org/licenses/by/4.0/), which permits unrestricted re-use, distribution, and reproduction in any medium, provided the original work is properly cited. 
processes with adverse consequences for an individual's future. Understanding the underlying risk factors for depression is therefore important, especially where such risk factors could be identified and modified.

The presence of an anxiety disorder is one such risk factor for the development of depression (Kessler et al. 1998; Wittchen et al. 2003a). Co-morbidity of anxiety disorders and depression is common (Kessler et al. 1996, 2005; Wittchen et al. 2003a, b). Kessler et al. (1996), using data from the original US National Comorbidity Survey, illustrated that the majority of cases of depression are secondary to another psychiatric diagnosis, with the primary disorder most commonly being an anxiety disorder. Anxiety disorders are often chronic in their course and frequently have onset in childhood or adolescence (Asselmann \& Beesdo-Baum, 2015). The general consensus from longitudinal studies in adolescents and in adults is that anxiety disorders precede depression more frequently than vice versa (Pine et al. 1998; Stein et al. 2001; Goodwin, 2002; Merikangas et al. 2003; Bittner et al. 2004).

However, diagnostic manuals (World Health Organization, 1993; American Psychiatric Association, 1994) suggest there are several distinct anxiety disorders. While most (e.g. social phobia, specific phobia, agoraphobia and post-traumatic stress disorder) must be linked to well-defined exposures, for generalized anxiety disorder (GAD) and panic disorder this is not the case. The cardinal symptom of GAD is chronic worry whereas a diagnosis of panic disorder requires the experience of recurrent panic attacks. GAD and panic disorder have distinct biology (Wilkinson et al. 1998), medical co-morbidities (Davies et al. 2012; Davies \& Allgulander, 2013) and differ in the frequency of reports of genetic association with depression (Roy et al. 1995; Kendler, 1996, Kendler et al. 2003, 2007). Factor analyses of cross-sectional data in large populations had suggested that GAD may be more closely associated with depression than is panic disorder (Krueger, 1999; Vollebergh et al. 2001; Slade \& Watson, 2006). Although this finding was confirmed in a population of adolescents and young adults (Beesdo-Baum et al. 2009), there remains a controversy over whether this factor structure is robust to addition of other diagnoses and in other age groups (Wittchen et al. 2009).

An analysis from the National Comorbidity Survey Replication reported correlations of 0.62 and 0.48 for 12-month prevalence of major depressive episode with GAD and panic disorder, respectively, in a population-based adult sample (Kessler et al. 2005). In a retrospective analysis of longitudinal data, in individuals who had met criteria for both GAD and major depressive episode (Wittchen et al. 2003b), the depression diagnosis followed that of GAD in $52 \%$ of individuals and preceded GAD in only $29 \%$. In a further study which relied on participants' recall in a population who had experienced depression and panic attacks, a first depressive episode was recalled as occurring after the first panic attack in $43 \%$ of cases and preceding it in $31 \%$ (Kessler et al. 1998). However, in a subsample who had had a diagnosis of panic disorder, which has a higher threshold, 22\% recalled that panic disorder preceded the first depressive episode whereas $48 \%$ reported that a depressive episode began first.

A limited number of longitudinal studies have attempted to investigate the risk that having an individual anxiety disorder or anxiety symptoms with onset in adolescence carries for subsequent development of depression. Some studies (e.g. Pine et al. 1998), having recruited individuals prior to adolescence and only examined anxiety disorder diagnoses specifically relevant to children, do not include disorders well recognized in adulthood such as GAD and panic disorder. However, a study of 2548 individuals aged 14-24 years, each followed up for 4 years (Bittner et al. 2004), provides some indication that GAD may have a higher predictive value for depression than does panic disorder. In unadjusted analyses all five of the anxiety disorders examined at baseline in this study, including GAD and panic disorder, conferred an elevated risk of first onset of major depressive disorder. However, after adjustment for prior co-morbid mental disorders, it appeared that panic disorder was no longer associated with subsequent depression. In contrast, an association of GAD with depression remained following adjustment, although there was considerable uncertainty as to the magnitude of the relationship as evidenced by the broad confidence intervals (CIs). Among individuals with GAD or agoraphobia at baseline, co-morbid panic attacks did not increase the risk for major depression. It should be noted, however, that in this study there was considerable heterogeneity of ages, with most of the sample being beyond adolescence even at entry (range 14-24 years). When the link between panic attacks and depression was examined after 10 years of follow-up, panic at baseline did confer an elevated risk for a diagnosis of depression over this longer period (Asselmann et al. 2014a).

A smaller study of 906 children aged 9-13 years reported no relationship between GAD and subsequent depression at ages 13-19 years (Bittner et al. 2007). The authors acknowledged that the predictive value of disorders may differ depending on the developmental stage. They postulated that the restrictive Diagnostic and Statistical Manual of Mental 
Disorders, 4th edition (DSM-IV) criteria for GAD in children and adolescents (American Psychiatric Association, 1994) made it more difficult to demonstrate associations with other psychiatric disorders. In contrast, GAD's diagnostic predecessor (overanxious disorder) did show an association with depression, as it had done in earlier studies (Pine et al. 1998). To our knowledge no study has specifically examined the impact of GAD and panic disorder from a fixed point in adolescence on the prevalence of depression at the onset of adulthood when the rise in incidence of depression is steepest.

While some evidence therefore suggests that GAD may be more closely associated with depression than is panic disorder, a single definitive mechanism that might underlie this link cannot be identified. Possible mechanisms which may contribute include:

(a) Genetics, as evidenced by the overlaps between GAD and depression discussed above. This might involve a genetic pleiotropy involving multiple polymorphisms predisposing to both depression and GAD simultaneously, one example being a polymorphism in the gene coding for the serotonin 5HT-1A receptor (Molina et al. 2011), although this has not been replicated in genome-wide association studies.

(b) A common biological mechanism such as cortisol dysregulation which is known to be involved in the aetiology of depression. A recent paper examining evidence of hypothalamic-pituitary-adrenal axis dysfunction across the anxiety disorders found evidence in GAD, but was inconclusive for panic disorder (Abelson et al. 2007; Faravelli et al. 2012).

(c) A vulnerability of GAD and depression, but not panic disorder to a specific but as yet unidentified risk factor (although a risk factor answering this description was not identified in an earlier examination of risk factors for depression and individual anxiety disorders; Beesdo et al. 2010).

(d) A psychological effect of one disorder leading to the other (e.g. generalized anxiety causing demoralization and leading to depression).

(e) GAD but not panic disorder being an early developmental manifestation of depression.

The Avon Longitudinal Study of Parents and Children (ALSPAC; Golding et al. 2001) provides an opportunity to study factors contributing to increased risk of depression at 18 years in a prospective cohort study design. In the present study we will consider symptoms of anxiety disorders in adolescence as risk factors for the subsequent development of depression by the age of 18 years, making the distinction between symptom patterns suggestive of GAD and panic disorder. Despite clear-cut differences in symptoms and diagnostic criteria, few studies have attempted to distinguish between the predictive ability of these two common forms of anxiety for the risk of future depression, which is important to know for the detection of depression and potentially for the prevention of its onset. Given the genetic associations between GAD and depression, and the suggestion of an association of these disorders at 4 years in an earlier longitudinal study, we hypothesized that GAD but not panic disorder symptoms at age 15 years are associated with a diagnosis of depression at 18 years. Elucidating this relationship may have practical implications for the prevention of depression.

\section{Method}

\section{Data source}

The sample comprised participants from the ALSPAC. All pregnant women resident in the former Avon Health Authority in south-west England having an estimated date of delivery between 1 April 1991 and 31 December 1992 were invited to take part. The children of 15247 pregnancies were recruited. Compared with the 1991 National Census data of residents in Avon, the ALSPAC had a slightly greater proportion of mothers who were married or cohabiting and who were owner-occupiers but was similar for other demographic variables. Ethical approval for the study was obtained from the ALSPAC Law and Ethics Committee and the Local Research Ethics Committees. More detailed information on the ALSPAC is available on the study website which contains details of all the data that are available through a fully searchable data dictionary (http://www.bris.ac.uk/alspac/research ers/data-access/data-dictionary/). Detailed information has been collected on the cohort since early pregnancy, including regular self-reported information from mothers and children and face-to-face assessments in research clinics (Boyd et al. 2013). The current study uses data from the remaining sample of ALSPAC offspring who attended the two most recent research clinics for the children at age 15 and 18 years.

\section{Sample size}

Our starting sample was those with complete anxiety and depression data from the Development and Well-Being Assessment (DAWBA; Goodman et al. 2000), the exposure variable, at age 15 years $(n=$ 5365). Those in the highest of the six bands of symptoms for depression on the DAWBA (indicating a probable diagnosis of depression based on symptoms over the past 2 weeks) at 15 years were removed from the sample at the outset $(n=89)$, including 40 out of 269 
$(15 \%)$ of those with the highest levels of GAD and four out of $32(13 \%)$ of those with the highest levels of panic symptoms. Of the remaining sample, 3635 adolescents also had complete depression outcome data using the Clinical Interview Schedule - Revised (CIS-R) at the age of 18 years. A final sample with complete data across all exposure, outcome and confounding variables comprised 2835 participants. However, all missing data were imputed and all analyses described below were repeated using the same starting sample size $(n=5365)$ in final sensitivity analyses.

We imputed for missing data because missing data can bias results if the data are not missing completely at random. Given that there is substantial information on sociodemographic variables in the ALSPAC that predict missingness, missing information can be assumed dependent on observed data. Thus we employed a fully conditional specification as implemented in the Multivariate Imputation by Chained Equations (MICE) algorithm in STATA 12, using all variables described in the analyses and additional auxiliary variables predictive of incomplete variables and/ or missingness (these included sociodemographic indicators and earlier continuous measures of depression; full list available on request) to predict missing data across 100 imputed datasets. The resulting Monte Carlo errors were less than $10 \%$ of the standard error and fraction of missing information (FMI) values were no larger than 0.4 , indicating that 100 imputed datasets were sufficient. Analyses were conducted post-imputation by combining estimates across imputed datasets using Rubin's rules.

\section{Measures}

$G A D$ and panic at age 15 years

Symptoms of GAD, panic and depression were measured by the DAWBA at the age of 15 years. We used the pencil and paper version of the DAWBA for self-completion. The DAWBA consists of questions about child mental health symptoms and their impact. The questions for each disorder follow the diagnostic criteria operationalized in the DSM-IV (American Psychiatric Association, 1994) or the International Classification of Diseases, 10th revision (ICD-10) Diagnostic Criteria for Research (World Health Organization, 1993). Accordingly, questions on GAD refer to the last 6 months and those on panic disorder to the last 4 weeks. Note that for GAD, the DAWBA employs the diagnostic criteria F93.80 from ICD-10's Diagnostic Criteria for Research, termed 'generalized anxiety disorder of childhood' which closely resembles the criteria for GAD in the DSM-IV (category 300.02) but differs markedly from ICD-10's adult GAD criteria (F41.1) which require the presence of autonomic symptoms and/or dry mouth. Each section contains 20-25 questions. Ordered categorical variables from 1 to 6 or 'bands' for each ICD-10 or DSM-IV disorder are derived from symptoms using a computerized algorithm (Goodman et al. 2011). This approach has the advantage of describing the symptom intensity for disorders along a continuum which may be particularly helpful in adolescence, where some disorders are rare but the presence of symptoms which do not meet the threshold for diagnosis is much more common. The levels of symptoms for each 'band' were chosen to provide an approximately evenly spaced progression in terms of the log odds of the child having the disorder in question (Goodman et al. 2011). Binary indicators of probable diagnosis are also generated. However, we used ordered-categorical measures in order to make full use of the symptom variation and to allow investigation of dose-response effects. Due to low frequency within some bands, we collapsed data from the original six levels to form three ordinal levels ranging from the lowest to highest probability of having the disorder in question (i.e. low, medium and high symptom bands). In addition, continuous symptom scores for GAD and panic were derived from the sum of all symptom items within the relevant section of the DAWBA. These symptom scores were used as exposure variables in secondary analyses.

\section{Depression at age 18 years (outcome variable)}

Depression at 18 years old was measured using the computerized version of the CIS-R. The CIS-R is a computerized interview that derives a diagnosis of depression according to ICD-10 criteria (World Health Organization, 1993), the time-frame being the past 2 weeks. The interview has been fully standardized and is equally reliable whether conducted by a lay or clinically trained interviewer or self-administered on the computerized version (Lewis, 1994). The CIS-R is designed for, and has been widely used within, community samples including the National Surveys of Psychiatric Morbidity and the 1958 birth cohort. A binary variable indicating presence $v$. absence of a diagnosis of depression was taken as the outcome measure.

\section{Confounding variables}

Variables that are associated with both anxiety and depression in adolescence were selected. These included maternal education (Letourneau et al. 2006; Chen \& Li, 2009; Park et al. 2013), social class (using the 'Standard Occupational Classification' of the Office of Population Censuses and Surveys, 1995), child gender, birth order (Gates et al. 1988), child smoking status (ever smoked a cigarette, yes/no) and frequency of 
child alcohol use (six-level ordinal variable from never to daily alcohol consumption).

\section{Analyses}

The exposure variable was DAWBA symptom bands at age 15 years (three-level ordered categorical variable) and the outcome was diagnosis of depression at age 18 years (binary variable). The associations between GAD and panic symptom bands and depression diagnosis were first investigated within separate logistic regression models using STATA 12 (StataCorp, 2011). We then investigated the association with both these exposures in the same logistic regression model. Confounding variables were then introduced into the models, including adjustments for depression symptoms at age 15 years (ordered categorical variable, using DAWBA bands from 1-5 with those in band 6 having been excluded). These analyses were repeated using symptom scores for GAD and panic as continuous exposure variables. Finally, the models described above were repeated following imputation for missing data. The population attributable fraction (PAF) was calculated as ( 1 - population unattributable fraction) using the 'punaf' command in STATA. This calculates the population unattributable fraction as the ratio of the log of scenario means for the outcome (depression at 18 years) in the baseline scenario $v$. the 'ideal' scenario in which the risk factor (here being medium or high anxiety at 15 years) is set to zero.

\section{Results}

\section{Descriptive statistics}

Sample demographics for the adolescents who comprised our complete case sample as compared with the rest of the ALSPAC are given in Table 1. As can be seen, the adolescents with complete case data came from more socially advantaged families.

\section{Co-morbidity}

Frequency of scoring within each of the DAWBA bands, co-morbidity and symptom scores are given in Tables 2 and 3. As can be seen, a large proportion of those with high levels of panic have high levels of GAD.

\section{Association between GAD and panic with confounding variables}

After removing those with co-morbid depression, those in higher GAD symptom bands at age 15 years were more likely to be girls $\left(\chi_{2}^{2}=182, p<0.001: 75 \%\right.$ of adolescents in the highest band were girls), to drink alcohol more frequently $\left(\chi_{12}^{2}=29, p<0.001\right)$ and to have ever smoked a cigarette $\left(\chi_{2}^{2}=14, p<0.001\right)$. A similar pattern was seen for panic: those in higher panic symptom bands were more likely to be girls $\left(\chi_{2}^{2}=27\right.$, $p<0.001$ : $75 \%$ of adolescents in the highest band were girls), to drink alcohol more frequently $\left(\chi_{12}^{2}=31\right.$, $p<0.001)$ and to have ever smoked a cigarette $\left(\chi_{2}^{2}=5\right.$, $p<0.084,68 \%$ of those in the top band compared with only $47 \%$ in the lowest band had tried a cigarette). There was no association between either GAD or panic and maternal education, social class or parity.

\section{Main analyses}

As can be seen in Table 4, adolescents with medium and high levels of either GAD or panic symptoms at the age of 15 years more frequently received a diagnosis of depression at 18 years. Univariate logistic regression models (Tables 5 and 6) provide strong evidence for an association between GAD and depression [odds ratio (OR) for high GAD symptom band $v$. low GAD symptom band $=6.6,95 \%$ CI 3.9-11.2] and a trend towards an association between panic and depression at age 18 years (OR for high panic symptom band $v$. low panic symptoms band $=3.1,95 \%$ CI $0.9-$ 10.8). The association of high GAD symptoms with depression was robust to the addition of panic (model 2) and to the addition of the six confounding variables described above (model 3, OR for high GAD symptom band $v$. low GAD symptom band $=5.2,95 \%$ CI 3.0-9.1) as well as the addition of baseline depression (model 4 , OR for high GAD symptom band $v$. low GAD symptom band $=3.8,95 \%$ CI 2.1-6.7). In contrast, the association with panic diminished markedly once GAD was included in the model (OR for high panic symptom band $v$. low panic symptom band $=1.6,95 \%$ CI 0.4-5.9) and remained so after adjustment for the further confounders and baseline depression. This reflects the relatively large proportion of those with high panic symptom scores also having high GAD symptom scores (32\%, see above). This pattern of results was also found when using the symptom scores as continuous exposure variables (Tables 5 and 6). The association between GAD and depression was strengthened following imputation for missing data, whilst any association with panic was further weakened (see Tables 7 and 8).

The PAF provides an index of the proportion of depression cases at 18 years that could be prevented by removing moderate or high symptoms of GAD at 15 years (assuming a causal relationship). Based on the fully adjusted regression (model 4) described above the PAF is $0.37(0.20-0.50)$, suggesting that, if causal, successfully eliminating moderate or high symptoms of GAD at 15 years would prevent $37 \%$ of depressed cases at 18 years. 
Table 1. Sample demographics

\begin{tabular}{|c|c|c|c|}
\hline & $\begin{array}{l}\text { ALSPAC sample lacking } \\
\text { exposure data, } n(\%)\end{array}$ & $\begin{array}{l}\text { Exposure (DAWBA) } \\
\text { data available, } n(\%)\end{array}$ & $\begin{array}{l}\text { Complete cases and depressed } \\
\text { cases at } 15 \text { years removed, } n(\%)\end{array}$ \\
\hline Participants, $n$ & 9613 & 5365 & 2835 \\
\hline Female & $6570(45)$ & $2820(53)$ & $1557(55)$ \\
\hline \multicolumn{4}{|l|}{ Maternal education } \\
\hline Up to 16 years only & $4808(70)$ & $2501(52)$ & $1347(48)$ \\
\hline Up to 18 years & $1402(20)$ & $1402(29)$ & $889(31)$ \\
\hline University degree & $723(10)$ & $887(19)$ & $599(21)$ \\
\hline \multicolumn{4}{|l|}{ Social class } \\
\hline Highest: 1 & $263(5)$ & $334(8)$ & $250(9)$ \\
\hline 2 & $1604(28)$ & $1581(36)$ & $1066(38)$ \\
\hline 3 & $3060(53)$ & $2060(47)$ & $1290(46)$ \\
\hline Lowest: 4 or 5 & $838(14)$ & $385(9)$ & $228(8)$ \\
\hline Mean age of mother, years (S.D.) & $26.7(5)$ & $28.5(5)$ & $28.9(4)$ \\
\hline First born & $4736(58)$ & $2518(51)$ & $1353(48)$ \\
\hline Never smoked (measured at age 15 years) & No data available & $2778(51.8)$ & $1613(56.9)$ \\
\hline Alcohol consumption & No data available & & \\
\hline Never & & $754(14.1)$ & $424(14.9)$ \\
\hline Once/twice & & $604(11.3)$ & $330(11.6)$ \\
\hline Used to drink but not now & & $166(3.1)$ & $79(2.8)$ \\
\hline Sometimes but less than once a week & & $2666(49.8)$ & $1458(51.4)$ \\
\hline 1-2 times a week & & $923(17.2)$ & $441(15.6)$ \\
\hline Drink more than twice a week & & $207(3.9)$ & $92(3.2)$ \\
\hline Drink every day & & $33(0.6)$ & $12(0.4)$ \\
\hline
\end{tabular}

ALSPAC, Avon Longitudinal Study of Parents and Children; DAWBA, Development and Well-Being Assessment; S.D., standard deviation.

Table 2. Mean total GAD symptom score according to each DAWBA symptom intensity band for GAD for the whole sample at age 15 years excluding those depressed $(\mathrm{n}=5276)$

\begin{tabular}{lccc}
\hline & Frequency, $n$ (\%) & $\begin{array}{l}\text { Mean GAD symptom } \\
\text { score (s.D.) }\end{array}$ & $\begin{array}{l}\text { Co-morbidity in high panic } \\
\text { symptom band, } n \text { (\%) }\end{array}$ \\
\hline Low GAD symptom band & $2096(40)$ & $0.9(0.8)$ & $1(0.1)$ \\
Medium GAD symptom band & $2951(56)$ & $2.8(1.6)$ & $18(0.6)$ \\
High GAD symptom band & $229(4)$ & $17.8(4.4)$ & $9(4)$ \\
\hline
\end{tabular}

GAD, Generalized anxiety disorder; DAWBA, Development and Well-Being Assessment, s.D., standard deviation.

${ }^{a}$ GAD measured by the DAWBA and divided into low, medium and high symptom bands (see the Method section).

Table 3. Mean total panic symptom score according to each DAWBA symptom intensity band for panic for the whole sample at age 15 years excluding those depressed $(\mathrm{n}=5276)$

\begin{tabular}{lccc}
\hline & Frequency, $n$ (\%) & $\begin{array}{l}\text { Mean panic symptom } \\
\text { score (s.D.) }\end{array}$ & $\begin{array}{l}\text { Co-morbidity in high GAD } \\
\text { symptom band, } n \text { (\%) }\end{array}$ \\
\hline Low panic symptom band & $5140(97)$ & $0.005(0.2)$ & $199(0.04)$ \\
Medium panic symptom band & $108(2)$ & $9.4(3.1)$ & $21(19)$ \\
High panic symptom band & $28(1)$ & $11.5(3.3)$ & $9(32)$ \\
\hline
\end{tabular}

DAWBA, Development and Well-Being Assessment, S.D., standard deviation; GAD, generalized anxiety disorder.

a Panic symptom intensity measured by the DAWBA and divided into low, medium and high symptom bands (see the Method section). 
Table 4. Frequencies of depression ${ }^{\mathrm{a}}$ at 18 years according to GAD and panic symptom severity ${ }^{\mathrm{b}}$ at 15 years by DAWBA symptom bands, for the non-depressed sample at 15 years $(\mathrm{n}=3635)$

Proportion (\%) of those in each symptom band at 15 years who go on to have depression $^{\mathrm{a}}$ at 18 years

Low GAD symptom band Medium GAD symptom band

$54 / 1433(4)$

High GAD symptom band

$163 / 1876(8)$

$38 / 124(23)$

Low panic symptom band

240/3544 (7)

Medium panic symptom band

$12 / 72(17)$

$3 / 16(17)$

GAD, Generalized anxiety disorder; DAWBA, Development and Well-Being Assessment; CIS-R, Clinical Interview Schedule - Revised.

${ }^{a}$ Depression identified by the CIS-R.

${ }^{\mathrm{b}}$ GAD and panic symptom intensity measured by the DAWBA and divided into low, medium and high symptom bands (see the Method section).

\section{Discussion}

Our findings confirm our original hypotheses that symptoms of GAD but not panic disorder at the age of 15 years are associated with depression at 18 years. Symptoms of GAD at 15 years are strongly associated with subsequent depression, and this relationship remained after adjustment for confounders including baseline depressive symptoms and was strengthened further on missing data imputation. The elevated risk for depression at 18 years applied even to those whose GAD symptoms at 15 years were rated as moderate. However, the apparent association between panic symptoms and later depression on the unadjusted analysis was a result of confounding by an association between panic and GAD. Overall, the present study strengthens the existing literature suggesting that depression is more closely associated with GAD than with panic disorder. It supports the broader concept that GAD and panic disorder differ in their co-morbidities.

We note that since lifetime incidence of depression prior to the age of 15 years was not assessed, these data do not allow us to say with certainty that GAD invariably precedes depression since it is conceivable that some participants had experienced depression earlier in their development which had remitted some time before their DAWBA at age 15 years. However given the relatively low prevalence of depression at 15 years we believe that such individuals are likely to be few in number. Despite an earlier analysis failing to identify temperament, personality or environmental risk factors shared by GAD and depression but not other anxiety disorder (Beesdo et al. 2010), there remains the possibility that our results reflect a developmental phenomenon, in that the expression of GAD symptoms could represent an early manifestation of depression if the two symptom groups had common aetiology through shared genetic, biological or environmental factors, or were manifestations of the same disorder. In some individuals the brain at 15 years may be more readily able to express anxiety, worry and the somatic symptoms of GAD than sadness, misery or the biological symptoms of depression despite the aetiology being the same. Thus development of GAD symptoms at 15 years could be seen as evidence of an individual being on a trajectory due to common underlying causes which will result in depression at 18 years unless adaptation occurs or treatment is instigated. However, some authors have argued that depression and GAD have sufficient differences in terms of risk factors, co-morbidities and treatment strategies that it is unrealistic to consider them as manifestations of a single underlying cause (Hettema, 2008). As such, it remains possible that the presence of generalized anxiety with pervasive worry causes demoralization which is more likely to lead to depression as a separate disorder, than is the more episodic phenomenon of panic attacks experienced in panic disorder.

Whether or not GAD and depression are truly separate disorders (Mennin et al. 2008), our study suggests that late adolescence is a period where GAD symptoms are common and are linked to an increased prevalence of depression subsequently. It is important to recognize the developmental context: the period from age 15 to 18 years is when the sharpest rise in the incidence of depression is observed, a disorder that has huge public health importance. Therefore it is possible that this period may provide an opportunity to identify and treat symptoms of GAD which is beneficial in itself and may have the added benefit of reducing the risk of a subsequent depressive disorder occurring. Identification and treatment of panic disorder remains important as this disorder is unpleasant and disabling in its own right. The lack of any association between panic symptoms and subsequent depression contrasts with the reported co-morbidity of panic disorder and depression in the general population (Skapinakis et al. 2011), suggesting that these are separate disorders that may co-occur without having a developmental link. However, it remains possible that depression following on from panic merely takes longer to emerge as suggested by reports of an association after 10 years' follow-up (Asselmann et al. 2014a) but not after 4 years (Bittner et al. 2004). Indeed, treatment of panic attacks in adolescents and young adults appeared to 
Table 5. OR for depression at 18 years according to GAD symptom band at age 15 years (compared with the lowest symptom band) and continuous symptom score in separate logistic regression models, combined models and following adjustments for confounding variables (models are for complete cases across all exposure, outcome and confounding variables with exclusion of those with depression at 15 years, $\mathrm{n}=2835)$

\begin{tabular}{|c|c|c|c|c|c|c|c|c|}
\hline & \multicolumn{2}{|c|}{$\begin{array}{l}\text { Model 1: risk for depression }{ }^{\text {a }} \\
\text { at } 18 \text { years, unadjusted }\end{array}$} & \multicolumn{2}{|c|}{$\begin{array}{l}\text { Model 2: risk for depression } \\
\text { at } 18 \text { years, adjusted for panic }\end{array}$} & \multicolumn{2}{|c|}{$\begin{array}{l}\text { Model 3: risk for depression } \\
\text { at } 18 \text { years, adjusted for } \\
\text { panic }^{b} \text { and confounders }\end{array}$} & \multicolumn{2}{|c|}{$\begin{array}{l}\text { Model 4: risk for depression } \\
\text { at } 18 \text { years, adjusted for } \\
\text { panic }^{\mathrm{b}} \text { and confounders }{ }^{\mathrm{c}}+ \\
\text { depression symptom bands } \\
\text { at } 15 \text { years }\end{array}$} \\
\hline & OR $(95 \% \mathrm{CI})$ & $p$ & OR $(95 \% \mathrm{CI})$ & $p$ & OR $(95 \% \mathrm{CI})$ & $p$ & OR $(95 \% \mathrm{CI})$ & $p$ \\
\hline \multicolumn{9}{|l|}{$\begin{array}{l}\text { ORs for depression at } 18 \text { years with GAD } \\
\text { symptom intensity }{ }^{\mathrm{b}} \text { at } 15 \text { years as exposure }\end{array}$} \\
\hline Low GAD symptom band & \multicolumn{2}{|l|}{1 (reference category) } & \multicolumn{2}{|l|}{1 (reference category) } & \multicolumn{2}{|c|}{1 (reference category) } & \multicolumn{2}{|c|}{1 (reference category) } \\
\hline Medium GAD symptom band & \multicolumn{2}{|l|}{$1.9(1.3-2.7)$} & \multicolumn{2}{|l|}{$1.9(1.3-2.7)$} & \multicolumn{2}{|c|}{$1.7(1.2-2.5)$} & \multicolumn{2}{|l|}{$1.5(0.9-2.1)$} \\
\hline High GAD symptom band & \multicolumn{2}{|l|}{$6.6(3.9-11.2)$} & \multicolumn{2}{|l|}{$6.3(3.7-10.8)$} & \multicolumn{2}{|l|}{$5.2(3.0-9.1)$} & \multicolumn{2}{|l|}{$3.8(2.1-6.7)$} \\
\hline Overall effect using likelihood ratio test & & $<0.0001$ & & $<0.0001$ & & $<0.0001$ & & 0.0001 \\
\hline Continuous GAD symptom score ${ }^{\mathrm{e}}$ & $1.5(1.4-1.7)$ & $<0.001$ & $1.5(1.4-1.7)$ & $<0.001$ & $1.5(1.3-1.7)$ & $<0.001$ & $1.4(1.2-1.6)$ & $<0.001$ \\
\hline
\end{tabular}

OR, Odds ratio; GAD, generalized anxiety disorder; CI, confidence interval; CIS-R, Clinical Interview Schedule - Revised; DAWBA, Development and Well-Being Assessment.

${ }^{a}$ Depression identified by the CIS-R

${ }^{\mathrm{b}} \mathrm{GAD}$ and panic symptom intensity at 15 years measured by the DAWBA and divided into low, medium and high symptom bands (see the Method section).

${ }^{\mathrm{C}}$ Social class, maternal education, birth order, child gender, child smoking status (ever smoked a cigarette, yes/no), child frequency of alcoholic drinks (six levels, 'I have never tried alcohol' up to 'I drink every day').

${ }^{\mathrm{d}}$ Depression symptom intensity at 15 years measured by the DAWBA, divided into bands $1-5$ (as described in the Method section, those in band 6 at age 15 years were excluded).

${ }^{\mathrm{e}}$ OR reflects increased odds for a 1 standard deviation symptom increase. 
Table 6. OR for depression at 18 years according to panic symptom severity at age 15 years (compared with the lowest symptom band) and continuous symptom score in separate logistic regression models, combined models and following adjustments for confounding variables (models are for complete cases across all exposure, outcome and confounding variables with exclusion of those with depression at 15 years; $\mathrm{n}=2835)$

\begin{tabular}{|c|c|c|c|c|c|c|c|c|}
\hline & \multicolumn{2}{|c|}{$\begin{array}{l}\text { Model 1: risk for depression }{ }^{\mathrm{a}} \\
\text { at } 18 \text { years, unadjusted }\end{array}$} & \multicolumn{2}{|c|}{$\begin{array}{l}\text { Model 2: risk for depression } \\
\text { at } 18 \text { years, adjusted for } \\
\text { GAD }\end{array}$} & \multicolumn{2}{|c|}{$\begin{array}{l}\text { Model 3: risk for depression } \\
\text { at } 18 \text { years, adjusted for } \\
\text { GAD }^{\mathrm{b}} \text { and confounders }\end{array}$} & \multicolumn{2}{|c|}{$\begin{array}{l}\text { Model 4: risk for depression } \\
\text { at } 18 \text { years, adjusted for } \\
\text { GAD }^{\mathrm{b}} \text { and confounders }{ }^{\mathrm{c}}+ \\
\text { depression symptom } \\
\text { bands }^{\mathrm{d}} \text { at } 15 \text { years }\end{array}$} \\
\hline & OR $(95 \% \mathrm{CI})$ & $p$ & OR $(95 \% \mathrm{CI})$ & $p$ & OR $(95 \% \mathrm{CI})$ & $p$ & OR $(95 \%$ CI $)$ & $p$ \\
\hline \multicolumn{9}{|l|}{$\begin{array}{l}\text { ORs for depression at } 18 \text { years with panic } \\
\text { symptom intensity }^{\mathrm{b}} \text { at } 15 \text { years as exposure }\end{array}$} \\
\hline Low panic symptom band & \multicolumn{2}{|l|}{1 (reference category) } & \multicolumn{2}{|l|}{1 (reference category) } & \multicolumn{2}{|c|}{1 (reference category) } & \multicolumn{2}{|c|}{1 (reference category) } \\
\hline Medium panic symptom band & \multicolumn{2}{|l|}{$2.1(0.9-5.0)$} & \multicolumn{2}{|l|}{$1.4(0.6-3.5)$} & \multicolumn{2}{|c|}{$1.4(0.6-3.5)$} & \multicolumn{2}{|c|}{$1.3(0.5-3.2)$} \\
\hline High panic symptom band & \multicolumn{2}{|l|}{$3.1(0.9-10.8)$} & \multicolumn{2}{|l|}{$1.6(0.4-5.9)$} & \multicolumn{2}{|l|}{$1.3(0.3-4.8)$} & $1.2(0.3-4.5)$ & \\
\hline Overall effect using likelihood ratio test & & 0.094 & & 0.629 & & 0.737 & & 0.838 \\
\hline Continuous symptom score ${ }^{\mathrm{e}}$ & $1.1(1.0-1.1)$ & 0.006 & $1.0(1.0-1.0)$ & 0.530 & $1.0(1.0-1.0)$ & 0.744 & $1.0(1.0-1.0)$ & 0.790 \\
\hline
\end{tabular}

OR, Odds ratio; GAD, generalized anxiety disorder; CI, confidence interval; CIS-R, Clinical Interview Schedule - Revised; DAWBA, Development and Well-Being Assessment.

${ }^{a}$ Depression identified by the CIS-R.

${ }^{\mathrm{b}} \mathrm{GAD}$ and panic symptom intensity at 15 years measured by the DAWBA and divided into low, medium and high symptom bands (see the Method section).

${ }^{\mathrm{c}}$ Social class, maternal education, birth order, child gender, child smoking status (ever smoked a cigarette, yes/no), child frequency of alcoholic drinks (six levels, ‘I have never tried alcohol' up to 'I drink every day').

${ }^{\mathrm{d}}$ Depression symptom intensity at 15 years measured by the DAWBA, divided into bands $1-5$ (as described in the Method section, those in band 6 at age 15 years were excluded).

${ }^{\mathrm{e}}$ OR reflects increased odds for a 1 standard deviation symptom increase. 
Table 7. Main analyses: model 3 before and after imputing missing data, according to GAD symptom band

\begin{tabular}{|c|c|c|c|c|}
\hline & \multicolumn{2}{|c|}{$\begin{array}{l}\text { Risk for depression }{ }^{\mathrm{a}} \text { at } 18 \text { years, } \\
\text { adjusted for panic }^{\mathrm{b}} \text { and } \\
\text { confounders }^{\mathrm{c}} \text {, complete cases } \\
(n=2835)\end{array}$} & \multicolumn{2}{|c|}{$\begin{array}{l}\text { Risk for depression }{ }^{\mathrm{a}} \text { at } 18 \text { years, } \\
\text { adjusted for panic }^{\mathrm{b}} \text { and } \\
\text { confounders }^{\mathrm{c}} \text {, after imputing for } \\
\text { missing data }(n=5276)\end{array}$} \\
\hline & OR $(95 \% \mathrm{CI})$ & $p$ & OR $(95 \%$ CI $)$ & $p$ \\
\hline Low GAD symptom band ${ }^{\mathrm{b}}$ & \multicolumn{2}{|c|}{1 (reference category) } & \multicolumn{2}{|c|}{1 (reference category) } \\
\hline Medium GAD symptom band ${ }^{b}$ & \multicolumn{2}{|c|}{$1.7(1.2-2.5)$} & \multicolumn{2}{|c|}{$2.0(1 .-2.7)$} \\
\hline High GAD symptom band ${ }^{\mathrm{b}}$ & \multicolumn{2}{|l|}{$5.2(3.0-9.1)$} & \multicolumn{2}{|l|}{$5.8(3.7-9.2)$} \\
\hline Continuous GAD symptom score ${ }^{\mathrm{d}}$ & $1.3(1.2-1.4)$ & $<0.001$ & $1.3(1.2-1.4)$ & $<0.001$ \\
\hline
\end{tabular}

OR, Odds ratio; CI, confidence interval; GAD, generalized anxiety disorder; CIS-R, Clinical Interview Schedule - Revised; DAWBA, Development and Well-Being Assessment.

${ }^{a}$ Depression identified by the CIS-R.

${ }^{\mathrm{b}} \mathrm{GAD}$ and panic symptom intensity at 15 years measured by the DAWBA and divided into low, medium and high symptom bands (see the Method section).

'Social class, maternal education, birth order, child gender, child smoking status (ever smoked a cigarette, yes/no), child frequency of alcoholic drinks (six levels, I have never tried alcohol up to I drink every day).

${ }^{\mathrm{d}}$ OR reflects increased odds for a 1 standard deviation symptom increase.

Table 8. Main analyses: model 3 before and after imputing missing data, according to panic symptom band

\begin{tabular}{|c|c|c|c|c|}
\hline & \multicolumn{2}{|c|}{$\begin{array}{l}\text { Risk for depression }{ }^{\mathrm{a}} \text { at } 18 \text { years, } \\
\text { adjusted for } \mathrm{GAD}^{\mathrm{b}} \text { and } \\
\text { confounders }{ }^{\mathrm{c}} \text {, complete cases } \\
(n=2835)\end{array}$} & \multicolumn{2}{|c|}{$\begin{array}{l}\text { Risk for depression }{ }^{\mathrm{a}} \text { at } 18 \text { years, } \\
\text { adjusted for } \mathrm{GAD}^{\mathrm{b}} \text { and } \\
\text { confounders }{ }^{\mathrm{c}} \text {, after imputing for } \\
\text { missing data }(n=5276)\end{array}$} \\
\hline & OR $(95 \% \mathrm{CI})$ & $p$ & OR $(95 \% \mathrm{CI})$ & $p$ \\
\hline Low panic symptom band ${ }^{b}$ & \multicolumn{2}{|c|}{1 (reference category) } & \multicolumn{2}{|c|}{1 (reference category) } \\
\hline Medium panic symptom band ${ }^{b}$ & \multicolumn{2}{|c|}{$1.4(0.6-3.5)$} & \multicolumn{2}{|l|}{$1.5(0.8-2.8)$} \\
\hline High panic symptom band ${ }^{\mathrm{b}}$ & \multicolumn{2}{|l|}{$1.3(0.3-4.8)$} & \multicolumn{2}{|l|}{$1.1(0.3-4.1)$} \\
\hline Continuous GAD symptom score ${ }^{\mathrm{d}}$ & $1.0(0.9-1.1)$ & 0.760 & $1.0(0.9-1.0)$ & 0.402 \\
\hline
\end{tabular}

OR, Odds ratio; CI, confidence interval; GAD, generalized anxiety disorder; CIS-R, Clinical Interview Schedule - Revised; DAWBA, Development and Well-Being Assessment.

${ }^{a}$ Depression identified by the CIS-R.

${ }^{\mathrm{b}} \mathrm{GAD}$ and panic symptom intensity at 15 years measured by the DAWBA and divided into low, medium and high symptom bands (see the Method section).

${ }^{\mathrm{c}}$ Social class, maternal education, birth order, child gender, child smoking status (ever smoked a cigarette, yes/no), child frequency of alcoholic drinks (six levels, I have never tried alcohol up to I drink every day).

${ }^{\mathrm{d}} \mathrm{OR}$ reflects increased odds for a 1 standard deviation symptom increase.

reduce the incidence of depression at the 10-year follow-up time point (Asselmann et al. 2014b).

GAD can be treated successfully, both by psychological therapies (Hunot et al. 2007) and by medications (Ravindran \& Stein, 2010; Baldwin et al. 2014). While the evidence is more limited for some drug classes in children than is the case in adults, cognitivebehavioural therapy is effective both in children and adolescents (Compton et al. 2004; Ishikawa et al.
2007). We hypothesize that successful treatment of adolescents with GAD could have the additional beneficial effect of reducing the risk of developing depression and its many undesirable consequences. Such an intervention could be considered to be a form of indicated prevention (Mrazek 2\& Haggerty, 1994). The impact of such an indicated prevention would need to be evaluated and balanced against any possible risk of self-harm (Olfson et al. 2003; Hammad, 2004). 
We advocate better mechanisms for identifying adolescents who experience GAD, including its core symptom of chronic worry. It is known that recognition rates for anxiety disorders in primary care are suboptimal (Wittchen et al. 2002, 2012). Although administration of screening instruments for anxiety disorders has been shown to be feasible in adults (Wittchen et al. 2002) and children (Simon \& Bögels, 2009) it is unclear whether it could be justified in primary care populations. A systematic review examining the value of screening for depression in primary care using a routinely administered questionnaire concluded that the benefits were marginal (Gilbody et al. 2005) although it was acknowledged that a more sophisticated twostage procedure for screening and case finding had yet to be evaluated.

The strengths of the study are the large sample size, that it is population-based, with both exposure and outcome data being collected from individuals at fixed ages, and the longitudinal design. Possible limitations include the attrition rates in the ALSPAC reducing representativeness and applicability of the sample to the general population. This problem was addressed at least in part by use of imputation techniques for missing data. While as many as $46 \%$ of individuals had one or more missing data value, the benefits of imputation are that biases associated with exclusion of subjects who do not provide complete data are avoided. As there was little difference between imputed and non-imputed results, our confidence in our findings is increased. The number of participants in the highest DAWBA symptom band for panic was small. The smaller number of participants in this symptom band reduces the power to find an association with depression at 18 years. The distribution of the panic symptom bands at 15 years reflects the fact that the panic disorder diagnosis is relatively rare at that age, but panic attacks are more common and our approach of using the DAWBA symptom bands as exposure variables rather than simply the presence or absence of a full clinical diagnosis mitigates the problem to some extent. Finally, the DAWBA estimates likelihood of diagnosis of GAD based on DSM-IV and the ICD-10 diagnostic criteria for research category F93.80. In both cases these criteria include symptoms such as sleep disturbance and poor concentration, which are also considered features of depression. Thus, using the DAWBA may tend to increase the overlap of GAD and depression compared with any instrument which used ICD's more autonomically oriented F41.1 criteria.

Further studies are required to establish whether identifying and treating GAD in adolescents reduces either the incidence or the severity of subsequent depression.

\section{Acknowledgements}

The UK Medical Research Council (grant reference74882), the Wellcome Trust (grant reference 076467) and the University of Bristol provide core support for the ALSPAC. The current project was supported by a Wellcome grant held by G.L. (084268/Z/ 07/Z). This article is the work of the authors, and S.J. C.D. and R.M.P. will serve as guarantor for the contents of this article and the analyses of data. We are extremely grateful to all the families who took part, the midwives for help in recruiting them, and the whole ALSPAC team, which includes interviewers, computer and laboratory technicians, clerical workers, research scientists, volunteers, managers, receptionists and nurses.

\section{Declaration of Interest}

None.

\section{References}

Abelson JL, Khan S, Liberzon I, Young EA (2007). HPA axis activity in patients with panic disorder: review and synthesis of four studies. Depression and Anxiety 24, 66-76.

American Psychiatric Association (1994). Diagnostic and Statistical Manual of Mental Disorders, 4th edn. American Psychiatric Association: Washington, DC.

Asselmann E, Beesdo-Baum K (2015). Predictors of the course of anxiety disorders in adolescents and young adults. Current Psychiatry Reports 17, 543.

Asselmann E, Wittchen HU, Lieb R, Höfler M, Beesdo-Baum K (2014a). Associations of fearful spells and panic attacks with incident anxiety, depressive, and substance use disorders: a 10-year prospective-longitudinal community study of adolescents and young adults. Journal of Psychiatric Research 55, 8-14.

Asselmann E, Wittchen HU, Lieb R, Höfler M, Beesdo-Baum K (2014b). Does helpseeking alter the risk for incident psychopathology in adolescents and young adults with and without fearful spells or panic attacks? Findings from a 10-year prospective-longitudinal community study. Journal of Affective Disorders 169, 221-227.

Baldwin DS, Anderson IM, Nutt DJ, Allgulander C, Bandelow B, den Boer H, Christmas DM, Davies S, Fineberg N, Malizia A, McCrone P, Nabarro D, O'Neill C, Scott J, van der Wee N, Wittchen H-U (2014).

Evidence-based pharmacological treatment of anxiety disorders, post-traumatic stress disorder and obsessivecompulsive disorder: a revision of the 2005 guidelines from the British Association for Psychopharmacology. Journal of Psychopharmacology 28, 403-439.

Beesdo K, Pine DS, Lieb R, Wittchen HU (2010). Incidence and risk patterns of anxiety and depressive disorders and categorization of generalized anxiety disorder. Archives of General Psychiatry 67, 47-57. 
Beesdo-Baum K, Höfler M, Gloster AT, Klotsche J, Lieb R, Beauducel A, Bühner M, Kessler RC, Wittchen HU (2009). The structure of common mental disorders: a replication study in a community sample of adolescents and young adults. International Journal of Methods in Psychiatric Research 18, 204-220.

Bittner A, Egger HL, Erkanli A, Jane Costello E, Foley DL, Angold A (2007). What do childhood anxiety disorders predict? Journal of Child Psychology and Psychiatry 48, 11741183.

Bittner A, Goodwin RD, Wittchen H-U, Beesdo K, Hofler M, Lieb R (2004). What characteristics of primary anxiety disorders predict subsequent major depressive disorders? Journal of Clinical Psychiatry 65, 618-626.

Boyd A, Goulding J, Macleod J, Lawler DA, Fraser A, Henderson J, Molloy L, Ness A, Ring S, Davey Smith G (2013). Cohort profile. The 'Children of the 90s' - the index offspring of the Avon Longitudinal Study of Parents and Children. International Journal of Epidemiology 42, 111-127.

Chen Y, Li H (2009). Mother's education and child health: is there a nurturing effect? Health Economics 28, 413-426.

Compton SN, March JS, Brent D, Albano A, Weersing VR, Curry J (2004). Cognitive-behavioral psychotherapy for anxiety and depressive disorders in children and adolescents: an evidence-based medicine review. Journal of the American Academy of Child and Adolescent Psychiatry 43, 930-959.

Davies S, Allgulander C (2013). Anxiety and cardiovascular disease. In Modern Trends in Pharmacopsychiatry - Anxiety Disorders, vol. 29 (ed. D. S. Baldwin and B. E. Lennard), pp. 85-97. Karger: Basel.

Davies SJC, Bjerkeset O, Nutt DJ, Lewis G (2012). A U-shaped relationship between systolic blood pressure and panic symptoms: the HUNT study. Psychological Medicine 42, 1969-1976.

Faravelli C, Lo Sauro C, Godini L, Lelli L, Benni L, Pietrini F, Lazzeretti L, Talamba GA, Fioravanti G, Ricca V (2012). Childhood stressful events, HPA axis and anxiety disorders. World Journal of Psychiatry 22, 13-25.

Gates L, Lineberger MR, Crockett J, Hubbard J (1988). Birth order and its relationship to depression, anxiety, and self-concept test scores in children. Journal of Genetic Psychology 149, 29-34.

Gilbody SM, House AO, Sheldon TA (2005). Screening and case finding for depression. Cochrane Database Systematic Reviews 2005, Issue 4. Art. No.: CD002792. doi:10.1002/ 14651858.CD002792.pub2.

Golding J, Pembrey M, Jones R, ALSPAC Study Team (2001). ALSPAC - the Avon Longitudinal Study of Parents and Children. I. Study methodology. Paediatric and Perinatal Epidemiology 15, 74-87.

Goodman A, Heiervang E, Collishaw S, Goodman R (2011). The 'DAWBA bands' as an ordered-categorical measure of child mental health: description and validation in British and Norwegian samples. Social Psychiatry and Psychiatric Epidemiology 46, 521-532.

Goodman R, Ford T, Richards H, Meltzer H (2000). The Development and Well-Being Assessment: description and initial validation of an integrated assessment of child and adolescent psychopathology. Journal of Child Psychology and Psychiatry 41, 645-655.

Goodwin RD (2002). Anxiety disorders and the onset of depression among adults in the community. Psychological Medicine 32, 1121-1124.

Hammad T (2004). Review and Evaluation of Clinical Data. Food and Drug Administration: Washington, DC (http:// www.fda.gov/ohrms/dockets/ac/04/briefing/2004-4065b110-TAB08-Hammads-Review.pdf). Accessed August 2015.

Hettema JM (2008). The nosologic relationship between generalized anxiety disorder and major depression. Depression and Anxiety 25, 300-316.

Hunot V, Churchill R, Teixeira V, Silva de Lima M (2007). Psychological therapies for generalised anxiety disorder. Cochrane Database of Systematic Reviews 2007, Issue 1. Art. No.: CD001848. doi:10.1002/14651858.CD001848.pub4.

Ishikawa SI, Okajima I, Matsuoka H, Sakano Y (2007). Cognitive behavioural therapy for anxiety disorders in children and adolescents: a meta-analysis. Child and Adolescent Mental Health 12, 164-172.

Jaffee SR, Moffitt TE, Caspi A, Fombonne E, Poulton R, Martin J (2002). Differences in early childhood risk factors for juvenile-onset and adult-onset depression. Archives of General Psychiatry 58, 215-222.

Kendler KS (1996). Major depression and generalised anxiety disorder. Same genes, (partly)differing environments revisited. British Journal of Psychiatry Supplement 30, 68-75.

Kendler KS, Gardner CO, Gatz M, Pedersen NL (2007). The sources of co-morbidity between major depression and generalized anxiety disorder in a Swedish national twin sample. Psychological Medicine 37, 453-462.

Kendler KS, Prescott CA, Myers J, Neale MC (2003). The structure of genetic and environmental risk factors for common psychiatric and substance use disorders in men and women. Archives of General Psychiatry 60, 929-937.

Kessler RC, Chiu WT, Dernier O, Walters EE (2005). Prevalence, severity, and comorbidity of 12-month DSM-IV disorders in the National Comorbidity Survey Replication. Archives of General Psychiatry 62, 617-627.

Kessler RC, Nelson CB, McGonagle KA, Liu J, Swartz M, Blazer DG (1996). Comorbidity of DSM-III-R major depressive disorder in the general population: results from the US National Comorbidity Survey. British Journal of Psychiatry Supplement 30, 17-30.

Kessler RC, Stang PE, Wittchen HU, Ustun TB, Roy-Burne PP, Walters EE (1998). Lifetime panic-depression comorbidity in the National Comorbidity Survey. Archives of General Psychiatry 55, 801-808.

Krueger RF (1999). The structure of common mental disorders. Archives of General Psychiatry 56, 921-926.

Letourneau NL, Fedick CB, Willms JD, Dennis C-L, Hegadoren K, Stewart MJ (2006). Longitudinal study of postpartum depression, maternal-child relationships, and children's behaviour to 8 years of age. In Parent-Child Relations: New Research (ed. D. Devore), pp. 45-63. Nova Science: New York.

Lewinsohn PM, Rohde P, Klein DN, Seeley JR (1999). Natural course of adolescent major depressive disorder: 
I. Continuity into young adulthood. Journal of the American Academy of Child and Adolescent Psychiatry 38, 56-63.

Lewis G (1994). Assessing psychiatric disorder with a human interviewer or a computer. Journal of Epidemiology and Community Health 48, 207-210.

Mennin DS, Heimberg RG, Fresco DM, Ritter MR (2008). Is generalized anxiety disorder an anxiety or mood disorder? Considering multiple factors we ponder the fate of GAD. Depression and Anxiety 25, 289-299.

Merikangas KR, He JP, Burstein M, Swanson SA, Avenevoli S, Cui L, Benjet C, Georgiades K, Swendsen J (2010). Lifetime prevalence of mental disorders in US adolescents: results from the National Comorbidity Study-Adolescent Supplement (NCS-A). Journal of the American Academy of Child and Adolescent Psychiatry 49, 980-989.

Merikangas KR, Zhang H, Avenevoli S, Acharyya S, Neuenschwander M, Angst J (2003). Longitudinal trajectories of depression and anxiety in a prospective community study: the Zurich Cohort Study. Archives of General Psychiatry 60, 993-1000.

Molina E, Cervilla J, Rivera M, Torres F, Bellón JA, Moreno B, King M, Nazareth I, Gutiérrez B (2011). Polymorphic variation at the serotonin 1-A receptor gene is associated with comorbid depression and generalized anxiety. Psychiatric Genetics 21, 195-201.

Mrazek P, Haggerty R (1994). Reducing Risks for Mental Disorders: Frontiers for Preventive Intervention Research. National Academies Press: Washington, DC.

Office of Population Censuses and Surveys (1995). Standard Occupational Classification, vol. 2, 2nd edn. HMSO: London.

Olfson M, Shaffer D, Marcus SC, Greenberg T (2003).

Relationship between antidepressant medication treatment and suicide in adolescents. Archives of General Psychiatry 60, 978-982.

Park AL, Fuhrer R, Quesnel-Vallée A (2013). Parents' education and the risk of major depression in early adulthood. Social Psychiatry and Psychiatric Epidemiology 48, 1829-1839.

Pine DS, Cohen P, Gurley D, Brook J \& Ma Y (1998). The risk for early-adulthood anxiety and depressive disorders in adolescents with anxiety and depressive disorders. Archives of General Psychiatry 55, 56-64.

Ravindran LN, Stein MB (2010). The pharmacologic treatment of anxiety disorders: a review of progress. Journal of Clinical Psychiatry 71, 839-854.

Roy MA, Neale MC, Pedersen NL, Mathé AA, Kendler KS (1995). A twin study of generalized anxiety disorder and major depression. Psychological Medicine 25, 1037-1049.

Simon E, Bögels SM (2009). Screening for anxiety disorders in children. European Child and Adolescent Psychiatry 18, 625-634.
Skapinakis P, Lewis G, Davies S, Brugha T, Prince M, Singleton N (2011). Panic disorder and subthreshold panic in the UK general population: epidemiology, comorbidity and functional limitation. European Psychiatry 26, 354-362.

Slade T, Watson D (2006). The structure of common DSM-IV and ICD-10 mental disorders in the Australian general population. Psychological Medicine 36, 1593-1600.

Sobocki P, Jonsson B, Angst J, Rehnberg C (2006). Cost of depression in Europe. Journal of Mental Health Policy and Economics 9, 87-98.

StataCorp. (2011). Stata Statistical Software: Release 12. StataCorp LP: College Station, TX.

Stein MB, Fuetsch M, Müller N, Höfler M, Lieb R, Wittchen HU (2001). Social anxiety disorder and the risk of depression: a prospective community study of adolescents and young adults. Archives of General Psychiatry 58, 251-256.

Vollebergh WA, Iedema J, Bijl RV, de Graaf R, Smit F, Ormel J (2001). The structure and stability of common mental disorders: The NEMESIS Study. Archives of General Psychiatry 58, 597-603.

Wilkinson DJ, Thompson JM, Lambert GW, Jennings GL, Schwarz RG, Jefferys D, Turner AG, Esler MD (1998). Sympathetic activity in patients with panic disorder at rest, under laboratory mental stress, and during panic attacks. Archives of General Psychiatry 55, 511-520.

Wittchen HU, Beesdo-Baum K, Gloster A, Höfler M, Klotsche J, Lieb R, Beauducel A, Bühner M, Kessler RC (2009). The structure of mental disorders re-examined: is it developmentally stable and robust against additions? International Journal of Methods in Psychiatric Research 18, 189-203.

Wittchen HU, Beesdo K, Bittner A, Goodwin RD (2003a). Depressive episodes - evidence for a causal role of primary anxiety disorders? European Psychiatry 18, 384-393.

Wittchen HU, Härtling S, Dukes E, Morlock R, Edelsberg J, Oster G, Berger A (2012). Generalized anxiety disorder in primary care. Patterns of healthcare utilization in Germany [article in German]. MMW Fortschritte der Medizin 154 (Suppl. 3), 77-84.

Wittchen HU, Kessler RC, Beesdo K, Krause P, Höfler M, Hoyer J (2002). Generalized anxiety and depression in primary care: prevalence, recognition, and management. Journal of Clinical Psychiatry 63 (Suppl. 8), 24-34.

Wittchen HU, Lecrubier Y, Beesdo K, Nocon A (2003b). Relationships among anxiety disorders: patterns and implications. In Anxiety Disorders (ed. D. J. Nutt and J. C. Ballenger), pp. 25-37. Blackwell Publications: Oxford.

World Health Organization (1993). The ICD-10 Classification of Mental and Behavioural Disorders: Diagnostic Criteria for Research. WHO: Geneva. 